\title{
Evidence on Physical Activity and the Prevention of Frailty and Sarcopenia Among Older People: A Systematic Review to Inform the World Health Organization Physical Activity Guidelines
}

\author{
Juliana S. Oliveira, Marina B. Pinheiro, Nicola Fairhall, Sarah Walsh, Tristan Chesterfield Franks, \\ Wing Kwok, Adrian Bauman, and Catherine Sherrington
}

\begin{abstract}
Background: Frailty and sarcopenia are common age-related conditions associated with adverse outcomes. Physical activity has been identified as a potential preventive strategy for both frailty and sarcopenia. The authors aimed to investigate the association between physical activity and prevention of frailty and sarcopenia in people aged 65 years and older. Methods: The authors searched for systematic reviews (January 2008 to November 2019) and individual studies (January 2010 to March 2020) in PubMed. Eligible studies were randomized controlled trials and longitudinal studies that investigated the effect of physical activity on frailty and/or sarcopenia in people aged 65 years and older. The Grading of Recommendations Assessment, Development and Evaluation approach was used to rate certainty of evidence. Results: Meta-analysis showed that physical activity probably prevents frailty (4 studies; frailty score pooled standardized mean difference, $0.24 ; 95 \%$ confidence interval, $0.04-0.43 ; P=.017, I^{2}=57 \%$, moderate certainty evidence). Only one trial investigated physical activity for sarcopenia prevention and did not provide conclusive evidence (risk ratio 1.08; 95\% confidence interval, $0.10-12.19$ ). Five observational studies showed positive associations between physical activity and frailty or sarcopenia prevention. Conclusions: Physical activity probably prevents frailty among people aged 65 years and older. The impact of physical activity on the prevention of sarcopenia remains unknown, but observational studies indicate the preventive role of physical activity.
\end{abstract}

Keywords: exercise, aging, frail elderly

Frailty and sarcopenia are common age-related conditions associated with adverse outcomes, such as falls, mobility disorders, the need for long-term care, and death. ${ }^{1,2}$ There is ongoing debate about how to best define frailty ${ }^{3}$ and sarcopenia. ${ }^{4}$

Frailty is characterized by a decline in functioning across multiple physiological systems that increases susceptibility to stressors. ${ }^{2}$ The concept of frailty generally involves a multidimensional condition that is part of the normal aging process. ${ }^{5}$ Frailty is dynamic, which means that the severity of frailty can change in either direction over time. ${ }^{2,5}$ Rockwood et $\mathrm{al}^{6}$ define frailty as a "multidimensional syndrome of loss of reserves (energy, physical ability, cognition, health) that gives rise to vulnerability." Fried et $\mathrm{al}^{7}$ developed the phenotype model to classify frailty, which consists of 5 physical components: unintentional weight loss, low grip strength, self-reported exhaustion, slow walking speed, and low physical activity. Frailty can be lessened by exercise-based interventions. ${ }^{8}$

Sarcopenia is a musculoskeletal disorder affecting older people that involves accelerated loss of muscle mass and function. ${ }^{1}$ Sarcopenia was defined by the European Working Group on Sarcopenia in Older People as (1) low muscle mass, (2) low muscle

Oliveira, Pinheiro, Fairhall, Kwok, and Sherrington are with the Institute for Musculoskeletal Health, The University of Sydney, Sydney, NSW, Australia; and the Sydney Local Health District, Camperdown, NSW, Australia. Oliveira, Pinheiro, Fairhall, Walsh, Chesterfield Franks, Kwok, and Sherrington are with the Sydney School of Public Health, Faculty of Medicine and Health, The University of Sydney, Sydney, NSW, Australia. Bauman is with the Prevention Research Collaboration, Sydney School of Public Health, The University of Sydney, Sydney, NSW, Australia. Oliveira (juliana.oliveira@sydney.edu.au) is corresponding author. strength, and/or (3) low physical performance..$^{9}$ The recent updated consensus statement from the European Working Group on Sarcopenia in Older People 2 defines sarcopenia as a "muscle disease (muscle failure) rooted in adverse muscle changes that accrue across a lifetime," notes that sarcopenia is common among adults of older age but can also occur earlier in life and focuses on "low muscle strength as a key characteristic of sarcopenia, uses detection of low muscle quantity and quality to confirm the sarcopenia diagnosis, and identifies poor physical performance as indicative of severe sarcopenia." 10 Sarcopenia is a clinical biomarker to identify people at increased risk of mobility disability. ${ }^{4}$ The Sarcopenia Definitions and Outcomes Consortium identified grip strength as an important discriminator of mobility disability and a predictor of health-related outcomes. ${ }^{4}$ Given the importance of strength and function in predicting adverse outcomes in older adults and the role of factors outside the muscle in strength and function (particularly neurological input), others have questioned the focus on muscle structure that is implied by sarcopenia and suggest use of terms such as muscle strength and motor impairment. ${ }^{11}$ It is well established that strength and function in older adults can be improved with exercise. ${ }^{2}$

Physical activity has also been identified as a potential preventive strategy to slow the development of both frailty and sarcopenia. We aimed to investigate evidence on the association between physical activity and prevention of frailty and sarcopenia in older people (aged $65 \mathrm{y}$ and older). Questions were: (1) What is the association between physical activity and sarcopenia/frailty prevention? (2) Is there a dose-response association (volume, duration, frequency, and intensity) between physical activity and the prevention of frailty and sarcopenia? and (3) Does the association vary by type or domain of physical activity? 


\section{Methods}

We conducted this review to inform the World Health Organization (WHO) Guideline Development Group in the update the WHO Global Recommendations on Physical Activity and Sedentary Behaviour (2020). The scope of the guideline, the Population, Intervention, Comparison, Outcome question, and the search strategy were decided by the Guideline Development Group. The Guideline Development Group initially requested an umbrella review (review of reviews). However, as we did not find any reviews, we extracted data from the individual studies that were reported in the reviews identified by the initial search strategy. We then conducted an updated and expanded search after submission of the report, to confirm that relevant studies were not missed. Therefore, this article includes the initial WHO report results as well as the updated search results.

\section{Search Strategy}

An initial search for systematic reviews was conducted in the PubMed database from 2008 to November 2019 (see Supplementary Material 1A [available online]). In addition, we conducted an updated and expanded search in PubMed from January 2010 to March 2020 (see Supplementary Material 1B [available online]). We also screened reference lists of relevant reviews and trials for additional studies. The language of publication was restricted to English.

Articles were independently screened in 2 stages: screening of title and abstracts and screening of full-text articles by 2 reviewers (J.S.O. and W.K. or S.W. and T.C.F) using the eligibility criteria (Supplementary Material 1C [available online]). Disagreements regarding the eligibility of studies were resolved through discussion and, when necessary, with the help of a third reviewer (C.S. or J.S.O). We conducted this systematic review following Preferred Reporting Items for Systematic Reviews and Meta-Analyses (PRISMA) guidelines, ${ }^{12}$ and the PRISMA study flow diagram documents the screening process.

\section{Selection Criteria}

We initially conducted a search for systematic reviews and metaanalyses. As we did not find any eligible systematic reviews, we identified reviews that included potentially eligible studies and screened all included studies against our questions. As there were too few individual studies investigating the effect of physical activity on the prevention of frailty and sarcopenia, we updated the search strategy to target the relevant studies that we could have missed.

In the updated/expanded review, we included randomized controlled trials (RCTs), prospective cohort studies, and retrospective cohort studies. We excluded cross-sectional, before-and-after studies, or non-RCTs.

\section{Type of Participants}

Studies conducted with participants aged 65 years and older were included. No restriction was placed on the source of the participants, that is, patients from primary care as well as community settings were eligible. We did not include studies in which more than $15 \%$ of the total sample had frailty or sarcopenia at baseline.

\section{Type of Interventions}

We included studies that involved physical activity of any type, mode, duration, frequency, or intensity. For the purpose of this review, physical activity encompasses any activity that requires energy expenditure such as walking, transport related, occupational, household chores, and structured exercises programs. ${ }^{13} \mathrm{We}$ excluded studies of multimodal interventions that did not present data on physical activity alone.

\section{Type of Outcome Measures}

The outcome was measures of frailty or sarcopenia status. We included only studies that specified sarcopenia and/or frailty were the outcome of interest, and we accepted the authors' definition of sarcopenia and/or frailty.

We used the Prevention of Falls Network Europe (ProFANE) taxonomy to classify the physical activity and exercise programs in the included studies (Supplementary Material 2 [available online]). The programs were classified as primarily involving the following exercise categories: (1) gait, balance, coordination, and functional task training (referred to as "balance and functional exercises" for simplicity); (2) strength/resistance training (including power training, using resistance so referred to as "resistance exercises"); (3) flexibility; (4) 3-dimensional (3D) exercise (with Tai Chi or dance subcategories); (4) general physical activity (walking programs); (5) endurance; (6) overall physical activity; and (7) other.

\section{Data Extraction}

Two independent reviewers (J.S.O. and W.K. or S.W. and T.C.F) extracted the characteristics and intervention outcomes of each trial according to the PRISMA Statement. ${ }^{14}$ The following data were extracted from each included trial: author, year of publication, country, sample characteristics (sample size, age and sex of participants, health status, and setting), intervention description (frequency, intensity, session duration, intervention duration, and who delivered the intervention), primary exercise according to ProFANE taxonomy, ${ }^{15}$ and methodological quality assessment using the Physiotherapy Evidence Database (PEDro) scale. We extracted frailty and sarcopenia status outcomes.

\section{Methodological Quality Assessment and Quality of the Evidence}

The PEDro scale was used to assess the internal validity of RCTs. ${ }^{16,17}$ The PEDro scale evaluates 11 items: inclusion criteria and source, random allocation, concealed allocation, similarity at baseline, subject blinding, therapist blinding, assessor blinding, completeness of follow-up, intention-to-treat analysis, betweengroup statistical comparisons, and point measures and variability. Scores on the PEDro scale range from 0 (very low methodological quality) to 10 (high methodological quality) and were downloaded from the PEDro database (https://www.pedro.org.au/). Methodological quality was not an inclusion criterion for this review.

We used the Grading of Recommendations Assessment, Development and Evaluation system to assess the overall certainty of the evidence ${ }^{18}$ for outcomes with results from 3 or more studies. We assigned a general rating of the certainty of the body of evidence as "High," "Moderate," "Low," or "Very low" based upon the presence or extent of 4 factors: risk of bias, inconsistency, imprecision, and publication bias. As we only included similar studies in terms of population, intervention, comparator, and outcome, we did not consider the indirectness criterion. 


\section{Data Analysis}

We performed meta-analyses with Comprehensive Meta-Analysis software (version 3; Biostat, Englewood, NJ) using the random effects model for frailty. We calculated treatment effects using standardized mean differences (Hedges $g$ ) standardized by postscore SD (or its estimate) with $95 \%$ confidence intervals (CIs). The standardized mean difference was calculated using the premean and postmean and SD or, when this was unavailable, using the mean change score. If multiple follow-up data points were provided, the scores obtained as close to the completion of the intervention as possible were used for the primary analysis. If the trial had more than 2 arms, we examined the overall effects of both arms separately (mean and SD). Effect sizes were categorized as small (0.2), medium (0.5), or large (0.8 or greater). We assessed funnel plot asymmetry visually and by using Egger test, with $P<.1$ considered as evidence of publication bias. For a study that only reported the number of sarcopenic participants in each group, we dichotomized the participants into 2 categories: nonsarcopenic and sarcopenic. We considered the occurrence of sarcopenia as the event, and we calculated risk ratio and $95 \% \mathrm{CI}$ using RevMan's calculator. ${ }^{19}$

\section{Results}

\section{Search}

The initial search for systematic reviews and meta-analyses did not identify enough evidence to answer the review question. We screened the full text of 34 reviews and did not find any eligible reviews (Figure 1). The main reasons for exclusion were reviews including younger participants $(\mathrm{n}=10)$, not including frailty-/ sarcopenia-related outcomes $(n=10)$, participants with frailty/sarcopenia at baseline (management instead of prevention, $n=6$ ), and multimodal interventions (eg, combined physical activity and nutrition intervention, $n=5)$. Among the 34 reviews which had their full text screened, 21 reviews included potentially eligible studies and their full texts were identified and assessed by 2 reviewers. We used the same eligibility criteria, but no restriction was applied for publication year of individual studies. We found 9 potential studies investigating the association between physical activity and the primary prevention of frailty/sarcopenia; however, frailty and sarcopenia status were an outcome in only 2 studies, which were included in the review. ${ }^{20,21}$

Our updated and expanded literature search identified a total of 1676 studies for the title and abstract screening, of which 172 were considered potentially eligible studies for full-text review. After the full-text screening, 8 eligible studies were identified and included in this review. ${ }^{20-29}$

We included a total of 10 studies in this review: 2 individual studies identified from the systematic reviews and 8 studies from the updated search. Figure 1 outlines the flow of studies through the review. We included $5 \mathrm{RCTs}^{20,22-24,29}$ and 5 longitudinal studies. $^{21,25-28,30}$ We pooled 4 included trials involving 1862 participants and 5 comparisons in the meta-analysis evaluating the effect of physical activity interventions on the prevention of frailty compared with control. ${ }^{20,22,29}$ One trial assessed the impact of physical activity on sarcopenia status outcome and involved a sample of 40 participants. $^{24}$

\section{Characteristics of Included Trials}

Publication dates ranged from 2013 to 2019 (median, 2017) with $40 \%$ of studies published after 2018. Studies were conducted in
8 different countries: single trials were conducted in China, ${ }^{22}$ Iceland, ${ }^{25}$ Taiwan, ${ }^{23}$ Spain, ${ }^{20}$ and Hong $\mathrm{Kong}^{21}$; 2 in Unites States, ${ }^{28,29}$ and 3 in Japan. ${ }^{24,26,27}$ Details of all included studies are summarized in Tables 1 and 2 .

\section{Quality}

Supplementary Table 1 (available online) summarizes the methodological quality and reporting of eligible trials. The total PEDro score ranged from 4 to 7 (mean of 6). Three trials were of high methodological quality on the PEDro scale $22,23,29$ (PEDro score $\geq 6$ ) and 2 trials were of low quality (PEDro score <6). ${ }^{20,24}$ All participants were randomly allocated and provided the calculation of point estimates and variability (PEDro items 2 and 11). Three trials out of $5(60 \%)$ did not report an intention-to-treat analysis. As expected in this type of intervention, none of the trials included blinded participants or blinded intervention deliverers.

\section{Participants}

In the studies reporting age, the mean age of participants ranged from 67 to 79 years. Nine studies recruited both men and women, ${ }^{20-25,27-29}$ and a single recruited only women. ${ }^{26}$ All participants from the included studies were recruited from the community. Four studies included prefrail participants at baseline, a single study involved a mix of frail and nonfrail, and 9 studies included a mix of presarcopenic and sarcopenic participants (see Tables 2 and 3).

\section{Outcome Measures}

Frailty was an outcome for 6 of the included studies. ${ }^{20,22,23,26}$ In 4 studies, ${ }^{20,22,23,26}$ frailty was measured using the following 5 Fried criteria: (1) unintentional weight loss, (2) exhaustion, (3) low physical activity, (4) slow walking speed, and (5) poor grip strength. Participants were classified as prefrail if they fulfilled 1 and 2 criteria, and frail if they fulfilled 3 or more criteria. A single study $^{29}$ measured frailty according to the SOF frailty index that included (1) inability to rise from a chair 5 times without using the arms, (2) self-reported reduced energy level, and (3) weight loss $\geq 4.55 \mathrm{~kg}$ or $\geq 5 \%$ during the last 12 months, or $\geq 2.275 \mathrm{~kg}$ or $\geq 2.5 \%$ during the last 6 months. Subjects were considered "frail" if at least 2 of the 3 criteria were fulfilled. One study ${ }^{28}$ measured frailty using gait speed and chair stand test. Frailty was indicated if (1) gait speed $<0.6 \mathrm{~m} / \mathrm{s}$ or (2) inability to rise from a chair without using one's arms.

Sarcopenia was an outcome in 4 studies. ${ }^{21,24,27,31}$ Two studies were defined according to the European Working Group on Sarcopenia in Older People. ${ }^{21,25,30}$ Participants who had (1) low muscle mass, (2) low muscle strength, and/or (3) low physical performance were classified as having sarcopenia. A single study ${ }^{27}$ measured sarcopenia by the estimation of appendicular lean mass/ height $^{2}$. One study ${ }^{24}$ assessed sarcopenia using Asia Working Group for Sarcopenia criteria.

\section{Exposure}

The included studies investigated different modalities of physical activity and exercise. Following the ProFANE taxonomy, a single study primarily investigated resistance training and 4 studies investigated exercise including multiple ProFANE categories. 


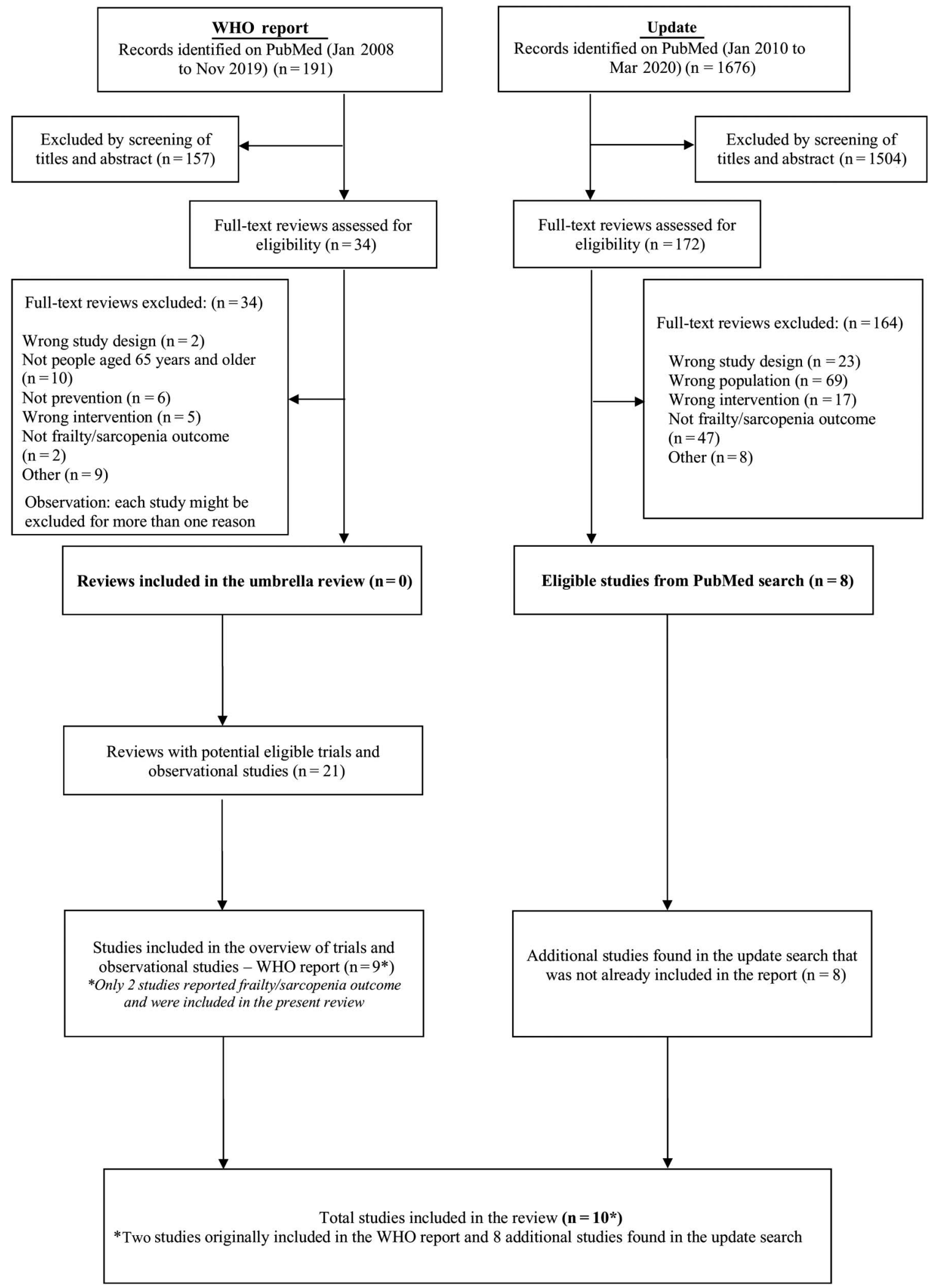

Figure 1 - Flow of studies through the review. 


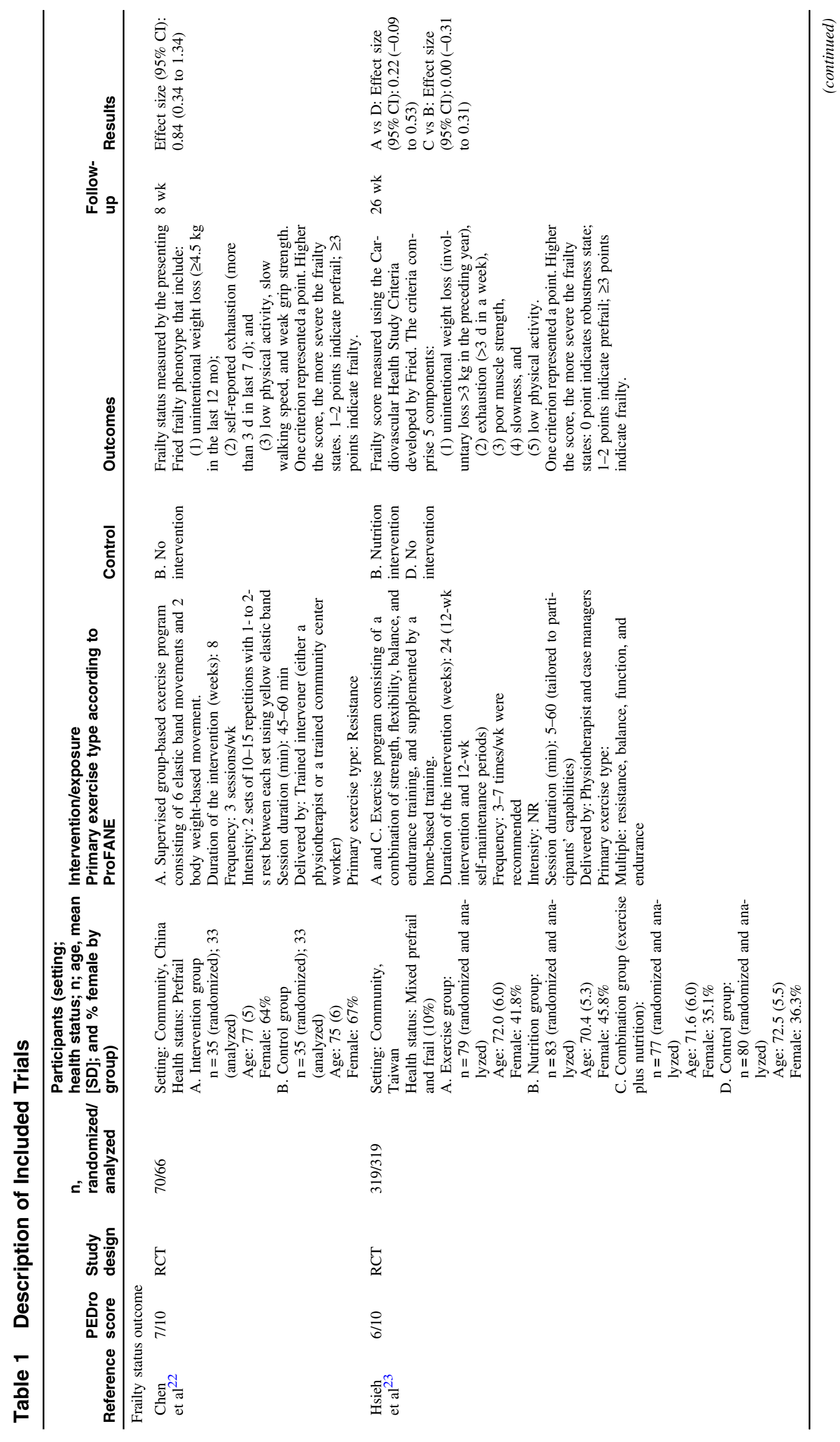




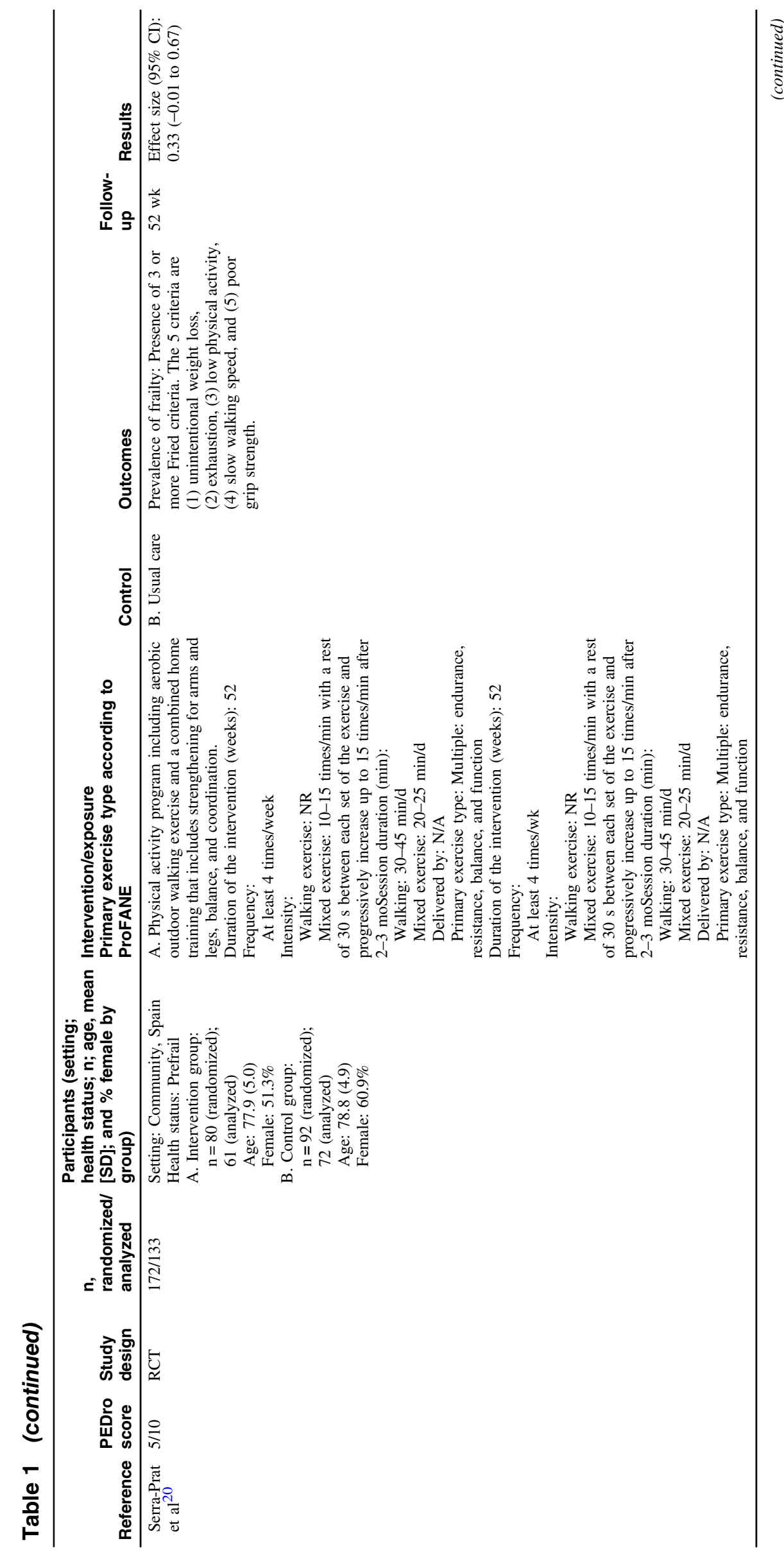




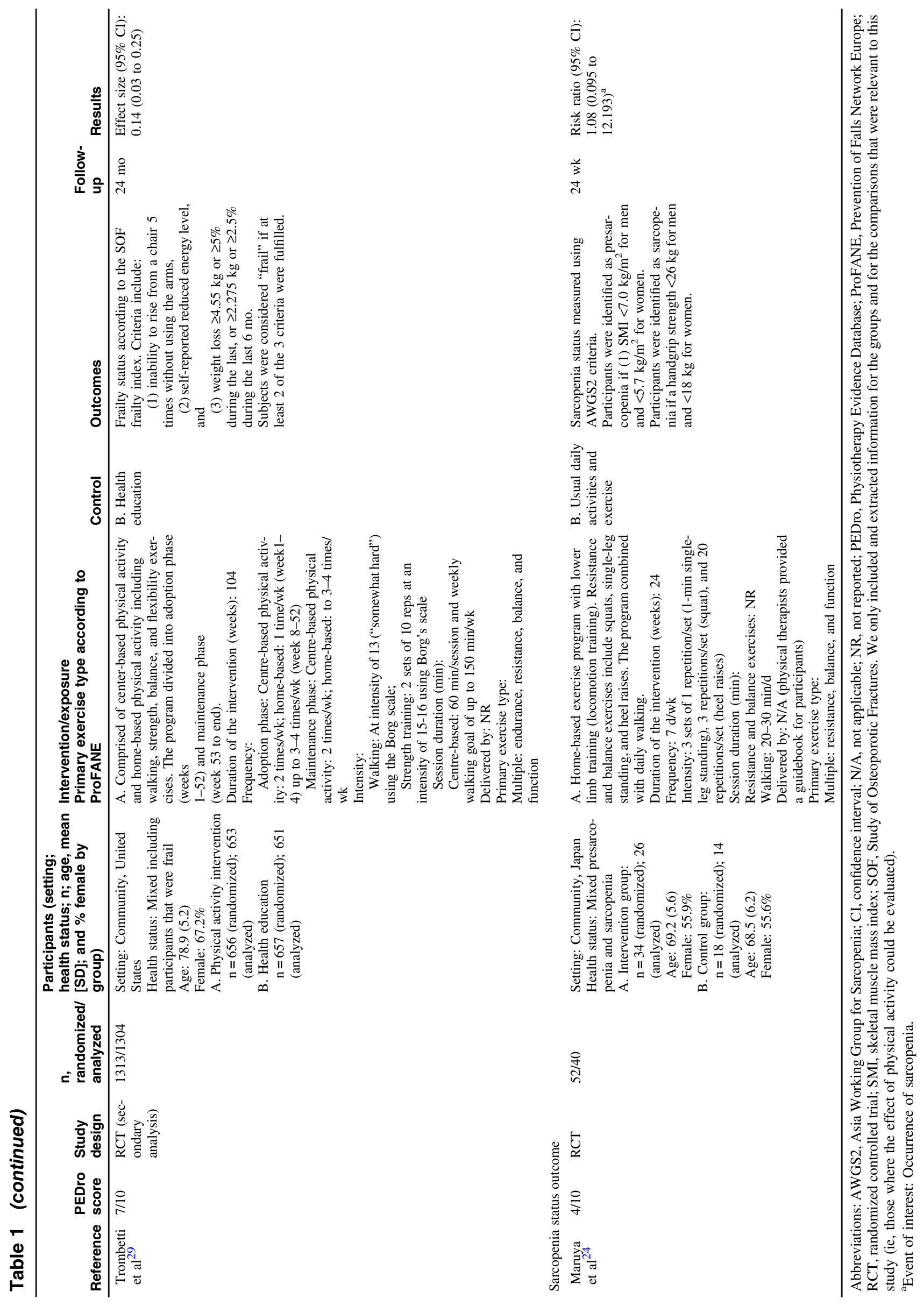




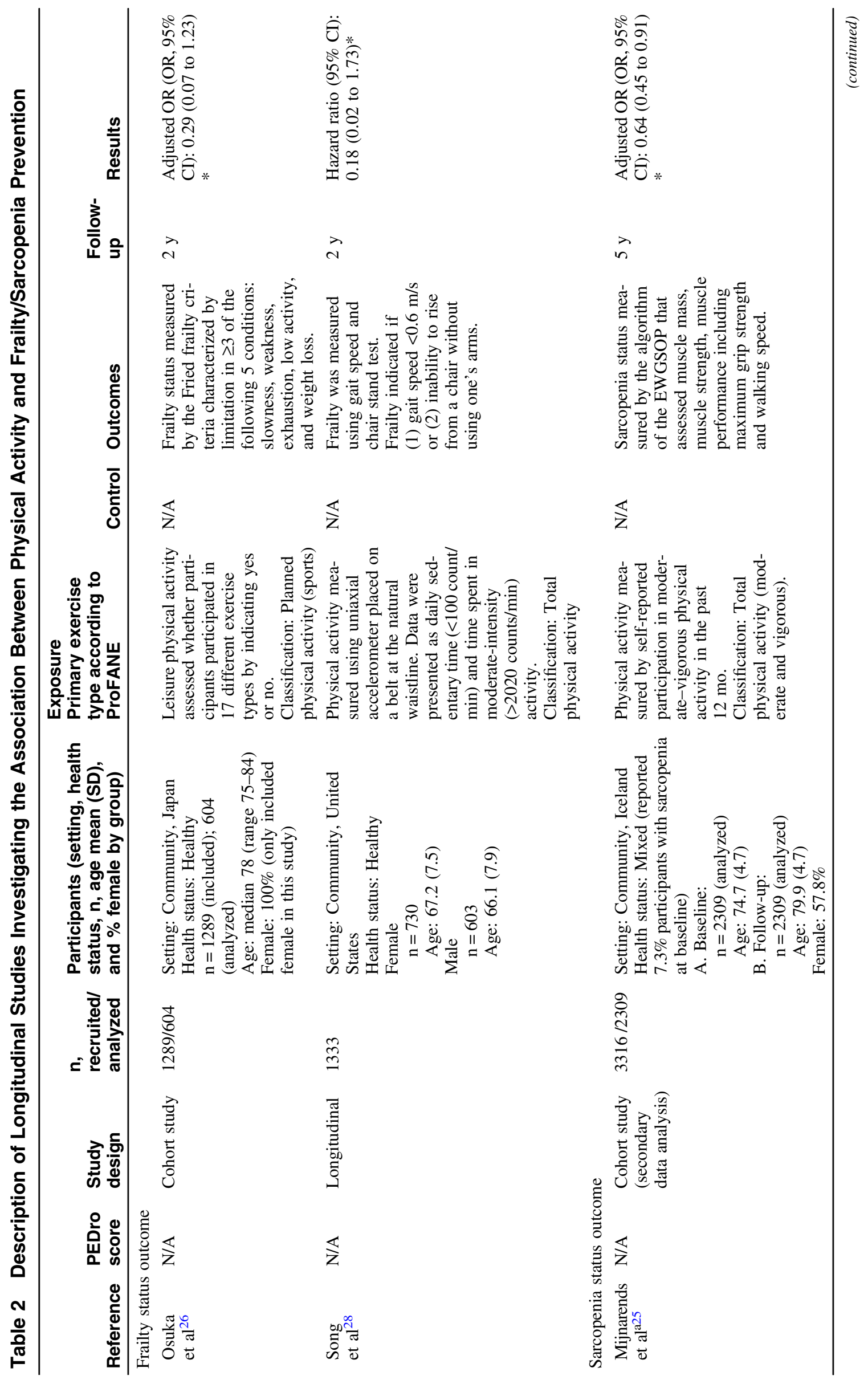




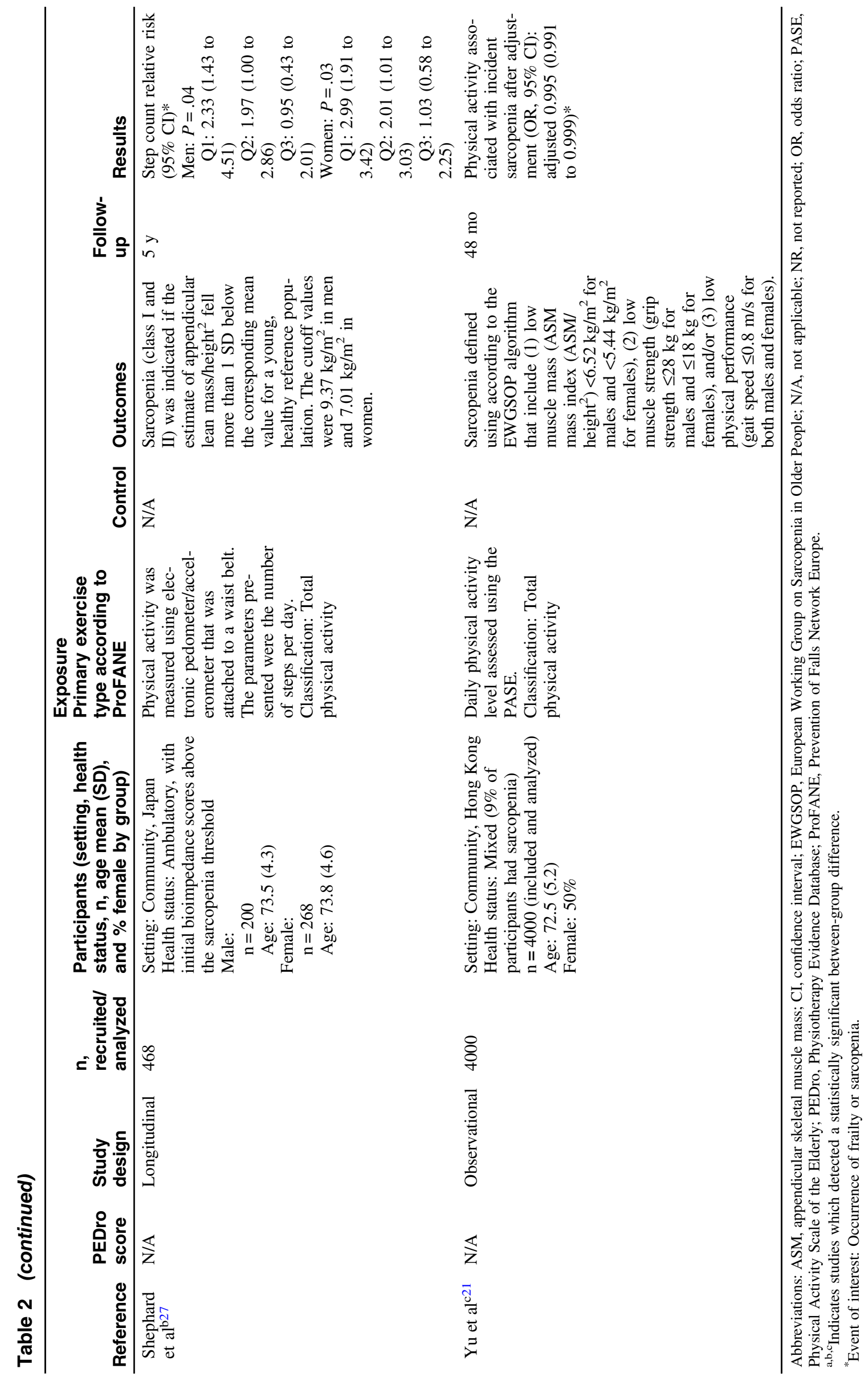




\section{Overview of Included Studies}

A total of $4 \mathrm{RCTs}^{20,22-24,29}$ investigated physical activity interventions compared with a control group. Overall, the sample size for the trials was reasonably large (median: 100, mean: 373, range: 40-1304) and the median follow-up length was 26 weeks. We identified 4 studies involving frailty outcomes and one study including sarcopenia outcome (Table 2).

The duration of the interventions investigating the effect of physical activity compared with control on frailty ranged from short (ie, $8 \mathrm{wk}$ ) to as long as 104 weeks, and the median duration was 38 weeks (mean $=47 \mathrm{wk}$ ). The intervention dose ranged from 3 to 7 sessions per week with the sessions ranging from 30 to 60 minutes. Only one trial investigated the effect of physical activity compared with control on sarcopenia. ${ }^{24}$ The duration of the intervention was 24 weeks and the frequency of the intervention was 7 times per week, including 20 to 30 minutes of supplemented walking exercise.

\section{Physical Activity and the Prevention of Frailty and Sarcopenia}

The pooled effect of physical activity intervention on frailty showed that physical activity significantly prevented frailty in intervention participants versus control participants ( 4 studies and 5 comparisons; frailty score standardized mean difference $=0.24$; $95 \%$ CI, 0.04 to $0.43 ; P=.017, I^{2}=57 \%$; Figure 2 ). The overall quality of evidence for the effect of physical activity on frailty was rated as "moderate quality". Details on the criteria used to apply the Grading of Recommendations Assessment, Development and Evaluation rating are provided in Table 3 and Supplementary Figure 1 (available online).

Only one trial reported the effect of physical activity intervention on sarcopenia outcome. This trial did not provide evidence of an association between physical activity and sarcopenia prevention (risk ration 1.08; 95\% CI, 0.10 to 12.19 ).

We included 5 observational studies, 2 studies investigated the association of physical activity with frailty prevention and 3 studies investigated the association with sarcopenia prevention. We were unable to perform meta-analysis due to the wide variation of design, statistical approach, and measures of physical activity across the studies. Each of these studies suggested a positive association between physical activity and prevention of frailty and sarcopenia (Table 3). The results in the 2 studies with frailty outcomes did not reach statistical significance, but there was statistically significant association between physical activity and the prevention of sarcopenia in each of the 3 studies with sarcopenia outcomes.

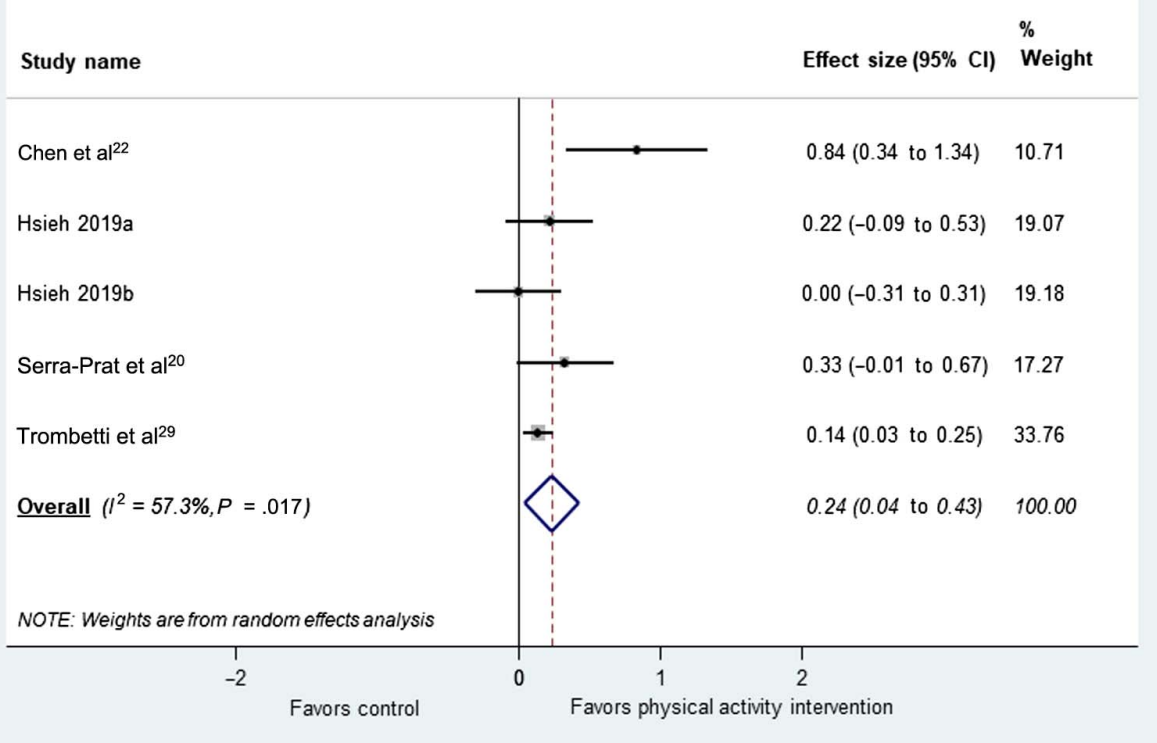

Figure 2 - Effect size $(95 \% \mathrm{CI})$ of physical activity interventions on frailty outcome by pooling data from 4 studies comparing physical activity versus control using random effects meta-analysis $(n=1862)$. CI indicates confidence interval.

Table 3 Summary of the Quality of Evidence and Strength of Recommendation

\begin{tabular}{|c|c|c|c|c|c|c|c|}
\hline \multirow[b]{2}{*}{ Outcome } & \multicolumn{2}{|c|}{ Summary of findings } & \multicolumn{5}{|c|}{ Quality of evidence assessment (GRADE) } \\
\hline & Effect size $^{\mathrm{a}}(95 \% \mathrm{Cl})$ & n (trials) & $\begin{array}{c}\text { Study } \\
\text { limitations }^{\text {b }}\end{array}$ & Imprecision $^{c}$ & Inconsistency ${ }^{d}$ & $\begin{array}{l}\text { Publication } \\
\text { bias }^{\mathrm{e}}\end{array}$ & Overall \\
\hline Frailty outcome & $0.24(0.04$ to 0.43$)$ & $1862(4)$ & None & None & $\downarrow$ & None & Moderate \\
\hline
\end{tabular}




\section{Dose-Response Associations, Type of Physical Activity}

We were unable to make clear statements about the optimal type of exercise or dose-response associations. Studies that detected impacts on relevant outcomes involved resistance training, combinations of resistance, balance and endurance exercise, as well as overall physical activity.

\section{Discussion}

We systematically reviewed the association between physical activity and the prevention of frailty and sarcopenia. Overall, we found that physical activity interventions may be effective in preventing frailty among people aged 65 years and older. The observational evidence also points toward beneficial associations between physical activity and frailty prevention. However, there was limited evidence evaluating preventive effects of physical activity on sarcopenia. As we only identified one trial evaluating the impact of physical activity on sarcopenia, no conclusion can be reached. This review was unable to draw firm conclusions on the modality of physical activity or the dose necessary to produce preventive benefits on frailty and sarcopenia. The results suggest that interventions involving resistance training and the combination of resistance, endurance, balance, and function probably have positive impact on frailty and sarcopenia; however, further investigation is warranted.

To the best of our knowledge, this is the first systematic review to evaluate the effectiveness of physical activity targeting the prevention of frailty and sarcopenia among people aged 65 years and older. Nonetheless, our study had several limitations. The initial aim of this review was to summarize the evidence of physical activity on prevention of frailty and sarcopenia in older people by conducting an umbrella review; however, we did not find any systematic reviews. We included the relevant studies that were in the reviews we found, and we conducted further searches to ensure that important studies were not missed. Furthermore, as the literature search was limited to PubMed and the English language, we may have missed relevant studies. Finally, another potential limitation is that we only included outcome data from the immediate postintervention time point, limiting our results to the short-term or immediate effect of physical activity on frailty and sarcopenia without consideration of the long-term effects.

We identified evidence of the effect of physical activity on frailty among people aged 65 years and older, but limited evidence on sarcopenia was found. There is a need for future research that evaluates the preventive impact of physical activity on sarcopenia. In addition, the optimal dose and type of physical activity for maximizing the preventive effect of physical activity on frailty sarcopenia is not clear, warranting further research. Furthermore, the longer of effect of physical activity on frailty and sarcopenia remains unknown, as our results were limited to the short-term effects. Studies that evaluate the long-term sustainability of physical activity behavior on frailty and sarcopenia are needed.

\section{Conclusion}

This review provides moderate certainty evidence that physical activity is an effective intervention for preventing frailty among people aged 65 years and older. We found limited evidence of any effect of physical activity on sarcopenia but promising results from observational studies. Further research is warranted to determine the impact, ideal dose, and modality of physical activity on frailty and sarcopenia. Future studies are also needed to explore the longterm sustainability of physical activity interventions.

\section{Acknowledgments}

The authors declare no conflicts of interest. This review was prepared for and funded by the World Health Organization, to contribute to the development of the Guidelines on Physical Activity and Sedentary Behaviour (2020). It was submitted to the Guideline Development Group and considered as they formulated their recommendations.

\section{References}

1. Cruz-Jentoft AJ, Sayer AA. Sarcopenia. Lancet. 2019;393(10191): 2636-2646. PubMed ID: 31171417 doi:10.1016/S0140-6736(19) 31138-9

2. Dent E, Martin FC, Bergman H, Woo J, Romero-Ortuno R, Walston JD. Management of frailty: opportunities, challenges, and future directions. Lancet. 2019;394(10206):1376-1386. PubMed ID: 31609229 doi:10. 1016/S0140-6736(19)31785-4

3. Rockwood K, Howlett SE. Fifteen years of progress in understanding frailty and health in aging. BMC Med. 2018;16(1):220. doi:10.1186/ s12916-018-1223-3

4. Cawthon PM, Travison TG, Manini TM, et al. Establishing the link between lean mass and grip strength cut-points with mobility disability and other health outcomes: proceedings of the sarcopenia definition and outcomes consortium conference. J Gerontol A Biol Sci Med Sci. 2019;75(7):1317-1323. PubMed ID: 30869772 doi:10.1093/ gerona/glz081

5. Xue QL. The frailty syndrome: definition and natural history. Clin Geriatr Med. 2011;27:1-15.

6. Rockwood K, Song X, MacKnight C, et al. A global clinical measure of fitness and frailty in elderly people. Can Med Assoc J. 2005; 173:489-495.

7. Fried LP, Tangen CM, Walston J, et al. Frailty in older adults: evidence for a phenotype. J Gerontol A Biol Sci Med Sci. 2001; 56(3):M146-M156. PubMed ID: 11253156 doi:10.1093/gerona/56. 3.M146

8. Cameron ID, Fairhall N, Langron C, et al. A multifactorial interdisciplinary intervention reduces frailty in older people: randomized trial. BMC Med. 2013;11(1):65. PubMed ID: 23497404 doi:10.1186/ 1741-7015-11-65

9. Cruz-Jentoft AJ, Baeyens JP, Bauer JM, et al. Sarcopenia: European consensus on definition and diagnosis: report of the European Working Group on sarcopenia in older people. Age Ageing. 2010; 39(4):412-423. PubMed ID: 20392703 doi:10.1093/ageing/afq034

10. Cruz-Jentoft AJ, Bahat G, Bauer J, et al. Sarcopenia: revised European consensus on definition and diagnosis. Age Ageing. 2019; 48(1):16-31. PubMed ID: 30312372 doi:10.1093/ageing/afy 169

11. Menant JC, Weber F, Lo J, et al. Strength measures are better than muscle mass measures in predicting health-related outcomes in older people: time to abandon the term sarcopenia? Osteoporos Int. 2017; 28(1):59-70. PubMed ID: 27394415 doi:10.1007/s00198-016-3691-7

12. Moher D, Liberati A, Tetzlaff J, Altman DG. Preferred reporting items for systematic reviews and meta-analyses: the PRISMA statement. BMJ. 2009;339:b2535. PubMed ID: 19622551 doi:10.1136/ bmj.b2535

13. World Health Organization. Global recommendations on physical activity for health. World Health Organization; 2010.

14. Liberati A, Altman DG, Tetzlaff J, et al. The PRISMA statement for reporting systematic reviews and meta-analyses of studies that 
evaluate healthcare interventions: explanation and elaboration. BMJ. 2009;339:b2700. doi:10.1136/bmj.b2700

15. Lamb SE, Becker C, Gillespie LD, et al. Reporting of complex interventions in clinical trials: development of a taxonomy to classify and describe fall-prevention interventions. Trials. 2011;12(1):125. PubMed ID: 21586143 doi:10.1186/1745-6215-12-125

16. Maher CG, Sherrington C, Herbert RD, Moseley AM, Elkins M. Reliability of the PEDro scale for rating quality of randomized controlled trials. Phys Ther. 2003;83(8):713-721. PubMed ID: 12882612 doi: $10.1093 / \mathrm{ptj} / 83.8 .713$

17. de Morton NA. The PEDro scale is a valid measure of the methodological quality of clinical trials: a demographic study. Aust J Physiother. 2009;55(2):129-133. PubMed ID: 19463084 doi:10.1016/ S0004-9514(09)70043-1

18. Guyatt GH, Oxman AD, Kunz R, et al. GRADE guidelines 6. Rating the quality of evidence-imprecision. J Clin Epidemiol. 2011;64(12):12831293. PubMed ID: 21839614 doi:10.1016/j.jclinepi.2011.01.012

19. Review Manager (RevMan). [computer program]. Version 5.3. Copenhagen, Denmark: The Nordic Cochrane Centre, The Cochrane Collaboration; 2014.

20. Serra-Prat M, Sist X, Domenich R, et al. Effectiveness of an intervention to prevent frailty in pre-frail community-dwelling older people consulting in primary care: a randomised controlled trial. Age Ageing. 2017;46(3):401-407. PubMed ID: 28064172 doi:10. 1093/ageing/afw242

21. Yu R, Wong M, Leung J, Lee J, Auyeung TW, Woo J. Incidence, reversibility, risk factors and the protective effect of high body mass index against sarcopenia in community-dwelling older Chinese adults. Geriatr Gerontol Int. 2014;14(suppl 1):15-28. PubMed ID: 24450557 doi:10.1111/ggi.12220

22. Chen R, Wu Q, Wang D, et al. Effects of elastic band exercise on the frailty states in pre-frail elderly people [published online ahead of print February 11, 2019]. Physiother Theory Pract. PubMed ID: 30741081 doi:10.1080/09593985.2018.1548673

23. Hsieh TJ, Su SC, Chen CW, et al. Individualized home-based exercise and nutrition interventions improve frailty in older adults: a randomized controlled trial. Int $J$ Behav Nutr Phys Act. 2019;16(1):119. PubMed ID: 31791364 doi:10.1186/s12966-0190855-9

24. Maruya K, Asakawa Y, Ishibashi H, Fujita H, Arai T, Yamaguchi H. Effect of a simple and adherent home exercise program on the physical function of community dwelling adults sixty years of age and older with pre-sarcopenia or sarcopenia. J Phys Ther Sci. 2016; 28(11):3183-3188. PubMed ID: 27942146 doi:10.1589/jpts.28.3183

25. Mijnarends DM, Koster A, Schols JM, et al. Physical activity and incidence of sarcopenia: the population-based AGES-Reykjavik Study. Age Ageing. 2016;45(5):614-620. PubMed ID: 27189729 doi:10.1093/ageing/afw090

26. Osuka Y, Kojima N, Yoshida Y, et al. Exercise and/or dietary varieties and incidence of frailty in community-dwelling older women: a 2-year cohort study. J Nutr Health Aging. 2019;23(5):425-430. PubMed ID: 31021359 doi:10.1007/s12603-019-1166-1

27. Shephard RJ, Park H, Park S, Aoyagi Y. Objectively measured physical activity and progressive loss of lean tissue in older Japanese adults: longitudinal data from the Nakanojo study. J Am Geriatr Soc. 2013;61(11):1887-1893. PubMed ID: 24219190 doi:10.1111/jgs.12505

28. Song J, Lindquist LA, Chang RW, et al. Sedentary behavior as a risk factor for physical frailty independent of moderate activity: results from the osteoarthritis initiative. Am J Public Health. 2015;105(7): 1439-1445. PubMed ID: 25973826 doi:10.2105/AJPH.2014.302540

29. Trombetti A, Hars M, Hsu FC, et al. Effect of physical activity on frailty: secondary analysis of a randomized controlled trial. Ann Intern Med. 2018;168(5):309-316. PubMed ID: 29310138 doi:10. 7326/M16-2011

30. Antoniak AE, Greig CA. The effect of combined resistance exercise training and vitamin D3 supplementation on musculoskeletal health and function in older adults: a systematic review and meta-analysis. BMJ Open. 2017;7(7):e014619. PubMed ID: 28729308 doi:10.1136/ bmjopen-2016-014619

31. Milder DA, Pillinger NL, Kam PCA. The role of prehabilitation in frail surgical patients: a systematic review. Acta Anaesthesiol Scand. 2018; 62(10):1356-1366. PubMed ID: 30094821 doi:10.1111/aas.13239 